\title{
Masks’ Effects on Recognizing Emotions During COVID-19
}

\author{
Betti (Zibei) Pang
}

The Baldwin School, Bryn Mawr, PA 19010.

Email: bettipang23@gmail.com

\begin{abstract}
The recognition of emotion with facial expressions is essential to human communication, with the eyes and mouth being the most prominent features to identify emotions. Masks have become a part of everyone's daily life during the pandemic. Since masks occlude the bottom half of the face, emotions may be more difficult to interpret accurately. The purpose of this study is to investigate the effects of face masks on the perception and recognition of emotions during COVID-19. For this study, a survey was created in which participants were asked to identify a person's facial expression, which was either unmasked or masked. The faces expressed 6 emotions: anger, disgust, fear, happiness, neutrality, and sadness. 35 total participants completed the survey. Overall, the answers for each emotion were more accurate with the unmasked faces than the masked faces. Happiness and neutrality had the highest percentage of correct answers in both unmasked and masked faces. Sadness had the highest percentage of incorrect answers in unmasked faces, while disgust had the highest percentage of incorrect answers in masked faces. Chi-squared tests were performed to see if the masked conditions were independent of emotion recognition accuracy. With all the emotions added together, the use of masks significantly affected the accuracy of the responses $(p<0.001)$. The responses for disgust $(p<0.001)$, fear $(p<0.05)$, happiness $(p<0.01)$, and sadness $(p<0.001)$ were significantly affected by the use of masks, while anger $(p>0.05)$ and neutrality $(\mathrm{p}>0.05)$ were not. In conclusion, this study found that the use of masks significantly affects the recognition of emotions, especially fear, happiness, sadness, and disgust.
\end{abstract}

Keywords: emotion perception, emotion recognition, emotional expression, masked emotions, facial expression recognition

\section{INTRODUCTION}

Over the past year and a half, COVID-19 has impacted lives all over the world.

Methods of communication have been affected, especially with the use of masks. As cities open up around the globe, masks continue to be daily essentials, impacting the communication of emotions. The expression of emotions can be visual, auditory, and tactile [1]. Specifically, visual expressions include facial expressions, which are an important part of nonverbal communication [2]. Since masks occlude the bottom half of the face, they may impact the recognition of emotions.

The recognition of emotion with facial expressions is significantly important in human communication. A lack of expressing and recognizing emotions can make social interactions more difficult, and may lead to isolation and physical or mental harm [3]. A 2017 study found that participants relied most strongly on the eyes and the mouth to recognize emotions [4]. Another previous study found that when identifying emotions, participants fixated on the eyes for the longest time, and then the nose and the mouth for a shorter amount of time [5]. This may indicate that the eyes play the most prominent role in identifying emotions. However, this study also found that participants needed to look at both the upper and lower half of the face to most accurately determine the emotion [5]. Additionally, another study found that occlusion of the mouth decreased recognition accuracy by more than $50 \%$ as compared to occlusion of the eyes [6]. This would mean that masks, which cover the nose and mouth, could impede the recognition of emotions.

The eyes and mouth may play a different role in the recognition of each emotion. It has been reported that anger, fear, and sadness rely more on the eyes, and happiness and disgust rely more on the mouth [4]. Additionally, another study found that disgust and 
surprise depended most on the eyes, while anger, fear, happiness, and sadness depended most on the mouth [6]. The emotions that depend more on the mouth will likely be more affected by a mask than emotions that rely on the eyes.

Masks only allow for the eyes and eyebrows to be seen, which may result in confusion and inaccurate recognition of expressions. Without a visible smile or frown, emotions may be unclear or seem less genuine [7]. Therefore, masks may result in the misidentification of emotions, affecting regular communication.

Currently, there have not been studies specifically aiming to find out how face masks affect emotion identification amid the COVID-19 pandemic. The purpose of this study is to investigate the effects of face masks on the perception and recognition of emotions during COVID-19.

Hypothesis: Recognition of emotions will be greatly impacted by mask wearing, especially with emotions that are more distinguishable by the mouth. Emotions that rely primarily on the expression of eyes will not be impacted as greatly.

\section{METHODS}

For this study, a survey was created in which participants were asked to identify a person's facial expression as one of the 6 emotions: anger, disgust, fear, happiness, neutrality, or sadness. Images of both unmasked and masked people were used to investigate the differences in accuracy.

\subsection{Faces and Expressions}

In the survey, the images of the various faces and emotions were taken from the FACES database [8]. In the whole dataset, each person expressed each emotion twice, resulting in an " $A$ " and " $B$ " set. From the " $A$ " data set, images of 6 different people expressing each of the 6 emotions were used, resulting in 36 different faces. The 6 different emotions were anger, disgust, fear, happiness, neutrality, and sadness. Using Adobe Photoshop, a mask was edited on each of the 36 images, to create a total of 72 images: 36 masked and 36 unmasked faces (Figure 1).

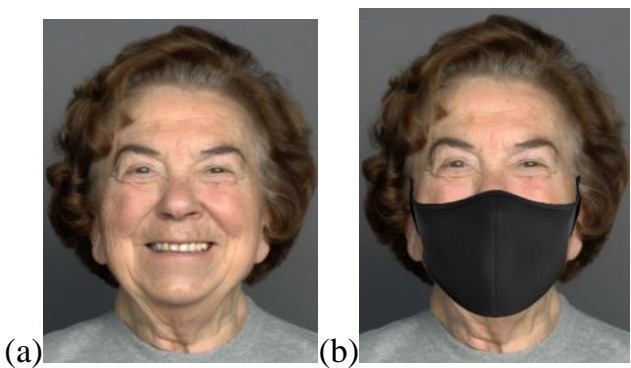

Figure 1. An image of a woman expressing happiness (a), and the same image but with a mask edited on top (b) [8].

\subsection{Survey Design}

The survey was made using Google Forms. The form included 3 sections, which were demographic questions, masked faces, and unmasked faces. In the demographic questions, participants were asked about their age range and gender. The masked faces section had 36 questions of the faces with masks, and the unmasked faces section had 36 questions of the faces without masks. The question order was randomized in each section, and the answers choices within each question were randomized as well.

To randomize the order of the last 2 sections, 2 versions of the form were created that had different orders of the masked and unmasked sections. Participants were told to follow instructions listed on a website made with Carrd (Figure 2). A link to a random number generator was provided, which randomly assigned the participant a "1" or a "2" [9]. Then, the participant was instructed to click on the corresponding version of the 2 forms (Figure 3).

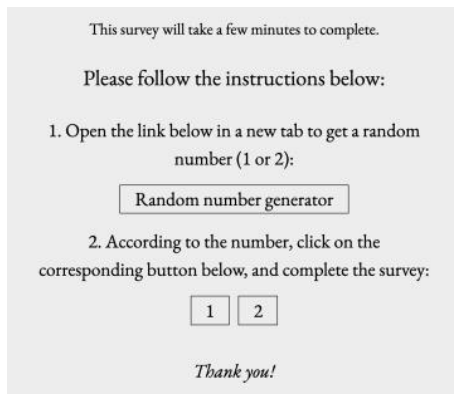

Figure 2. The Carrd website with instructions to complete the survey.
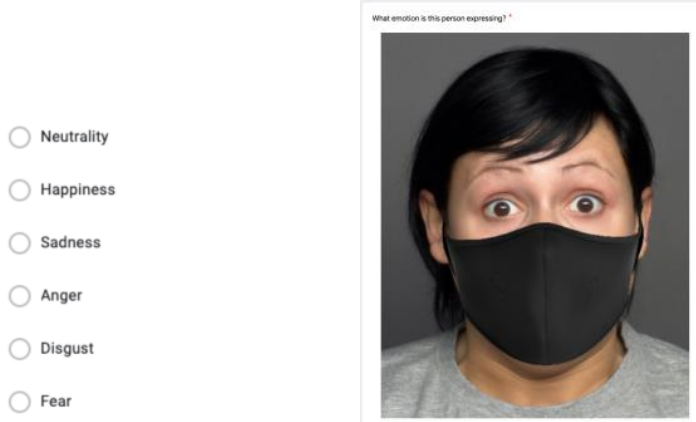

Figure 3. An example question from the survey that shows a masked face expressing fear.

\subsection{Statistics}

Chi-square tests were done using $\mathrm{R}$ language (RStudio v4.1.1) (Figure 4).

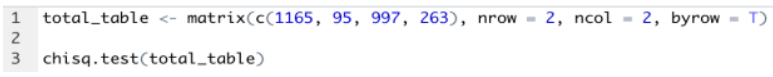

Figure 4. The R code for a chi-square test. 


\section{RESULTS}

\subsection{Participants}

35 total participants completed the survey; 16 completed the survey that had the unmasked faces first, and 19 completed the survey that had the masked faces first. Any differences in responses between the 2 versions of the form were not significant $(\mathrm{p}>0.05) .33$ of the participants were between ages 14-18, 1 participant was between ages 9-13, and another participant was between ages 19-23. 27 participants were women, 1 was a man, 4 were nonbinary, and 1 was genderfluid.

\subsection{Masked Condition and Accuracy}

The overall number of emotions that were correctly identified, masked or unmasked, was calculated by adding together all of the correct answers for each different emotion. The total number of 420 is the highest possible number of correctly answered questions. Disgust and sadness had the lowest number of correct responses, while happiness had the highest number of correct responses (Table 1).

Table 1. The number and percent of responses with correctly identified emotions.

\begin{tabular}{llll}
\hline & Total \# & \# Correct & \% Correct \\
\hline Anger & 420 & 369 & $87.9 \%$ \\
Disgust & 420 & 294 & $70 \%$ \\
Fear & 420 & 394 & 93.8 \\
Happiness & 420 & 408 & $97.1 \%$ \\
Neutrality & 420 & 398 & $94.8 \%$ \\
Sadness & 420 & 299 & $71.2 \%$ \\
\hline
\end{tabular}

The total number of correct answers for each emotion were then separated into whether the face was unmasked or masked (Table 2). The highest possible number of unmasked or masked correct answers was 210. Disgust and sadness had the lowest number of correct responses, while happiness had the highest number of correct responses. Disgust had the largest difference in accuracy percentage between the masked and unmasked (Figure 5).

Table 2. The number and percent of responses with correctly identified masked or unmasked emotions.

\begin{tabular}{lllll}
\hline & $\begin{array}{l}\text { Unmas } \\
\text { ked }\end{array}$ & $\begin{array}{l}\text { Mas } \\
\text { ked }\end{array}$ & $\begin{array}{l}\text { \% Correct } \\
\text { Unmasked }\end{array}$ & $\begin{array}{l}\text { \% Correct } \\
\text { Masked }\end{array}$ \\
\hline Anger & 189 & 180 & $90 \%$ & $85.7 \%$
\end{tabular}

\begin{tabular}{lcccc} 
Disgust & 196 & 98 & $93.3 \%$ & $46.7 \%$ \\
Fear & 203 & 191 & $96.7 \%$ & $91.0 \%$ \\
Happiness & 210 & 198 & $100 \%$ & $94.3 \%$ \\
Neutrality & 200 & 198 & $95.2 \%$ & $94.3 \%$ \\
Sadness & 167 & 132 & $79.5 \%$ & $62.9 \%$ \\
\hline
\end{tabular}

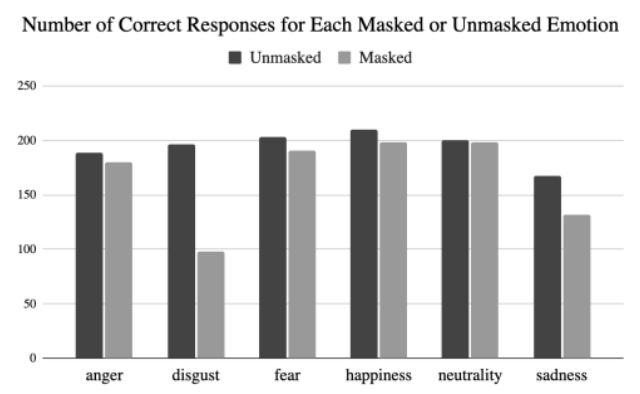

Figure 5. The number of correct responses for each emotion, with a masked or unmasked face.

Then, the total number of unmasked or masked emotions was separated into whether the emotion was correctly or incorrectly recognized by the participants. Overall, the answers for each emotion were more accurate with the unmasked faces than the masked faces. Sadness had the highest percentage of incorrect unmasked answers, while disgust had the highest percentage of incorrect masked answers. Happiness and neutrality had the least incorrect answers in both unmasked and masked faces (Figure 6).

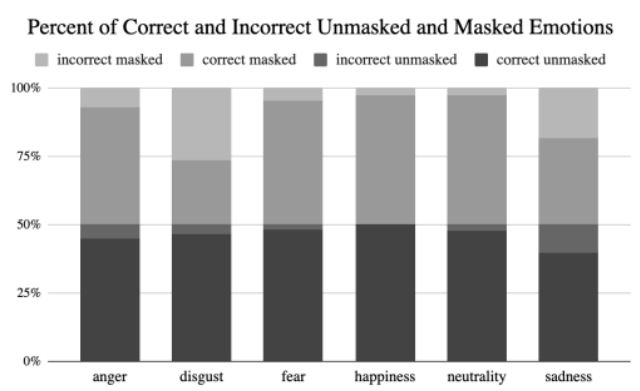

Figure 6. The percent of correct and incorrect responses with a masked or unmasked face, with each emotion.

\subsection{Chi-squared Tests}

Chi-squared tests were performed to see if the masked conditions were independent of emotion recognition accuracy. With all the emotions added together, the use of masks significantly affected the accuracy of the responses $(p<0.001)$. Individually, disgust $(p<0.001)$, fear $(\mathrm{p}<0.05)$, happiness $(\mathrm{p}<0.01)$, and sadness $(\mathrm{p}<0.001)$ were all significantly affected by the use of masks, while 
anger $(\mathrm{p}>0.05)$ and neutrality $(\mathrm{p}>0.05)$ were not (Table 3).

Table 3. Pearson's Chi-squared test (with Yates' continuity correction) of each emotion, comparing accuracy with masked condition.

\begin{tabular}{llll}
\hline & $\chi^{\wedge} 2$ & df & p-value \\
\hline Anger & 1.4283 & 1 & 0.232 \\
Disgust & 106.68 & 1 & $<2.2 \mathrm{e}-16$ \\
Fear & 4.961 & 1 & 0.02593 \\
Happiness & 10.38 & 1 & 0.001274 \\
Neutrality & 0.047967 & 1 & 0.8266 \\
Sadness & 13.42 & 1 & 0.000249 \\
Total & 90.802 & 1 & $<2.2 \mathrm{e}-16$ \\
\hline
\end{tabular}

\section{DISCUSSION}

\subsection{Emotions Affected Significantly}

\subsubsection{Disgust}

This study showed that recognition of disgust is significantly affected by masks $(\mathrm{p}<2.2 \mathrm{e}-16)$, which suggests that the expression of disgust heavily relies on the lower half of the face. Wegrzyn and others found that disgust has a stronger reliance on the mouth [4]. However, according to Kotsia and others, disgust has a stronger reliance on the eyes [6]. Similarly to fear and sadness, disgust may have been confused with similar expressions for other negative emotions, especially anger.

\subsubsection{Sadness}

The data in this study indicated that recognition of sadness is significantly affected by mask usage ( $\mathrm{p}=$ 0.000249), which suggests that the expression of sadness greatly relies on the lower half of the face. According to Kotsia and others, sadness has a stronger reliance on the mouth [6]. However, Wegrzyn and others found that sadness has a stronger reliance on the eyes [4]. Similarly to fear, sadness may have been confused with other negative emotions, like disgust, fear, and anger.

\subsubsection{Happiness}

This study also showed that recognition of happiness is significantly affected by masks $(p=0.001274)$, which suggests that the expression of happiness largely relies on the lower half of the face. Kotsia and others also found that happiness has a stronger reliance on the mouth [6]. Additionally, Wegrzyn and others also found that happiness has a stronger reliance on the mouth [4]. These results align with the fact that happiness is characterized by a smile, which appears most prominently in the mouth. Additionally, as the only key positive emotion, it is less likely to be confused with other expressions.

\subsubsection{Fear}

From the data found in this study, the recognition of fear is significantly affected by masks $(p=0.02593)$, which suggests that the expression of fear greatly relies on the lower half of the face. Kotsia and others also found that fear has a stronger reliance on the mouth [6]. However, according to Wegrzyn and others, fear has a stronger reliance on the eyes [4]. With only the eyes visible, fear may have been confused with other negative emotions, like anger, disgust or sadness.

\subsection{Emotions Not Affected Significantly}

\subsubsection{Anger}

This study indicated that recognition of anger is not significantly affected by the usage of masks $(p=0.232)$, which suggests that the expression of anger relies more on the upper half of the face. Wegrzyn and others found that anger has a stronger reliance on the eyes, but more commonly in the male face [4]. However, according to Kotsia and others, anger has a stronger reliance on the mouth [6]. Similarly to the other negative emotions, anger may have been confused with similar negative expressions.

\subsubsection{Neutrality}

The data in this study showed that recognition of neutrality is not significantly affected by masks ( $p=$ 0.8266), which suggests that the expression of neutrality does not rely heavily on the lower half of the face. However, Wegrzyn and others found that anger has a stronger reliance on the mouth, but more commonly in the male face [4]. Neutrality may have had varied results because individuals have slightly different resting faces.

\subsection{Limitations}

The participants in this study were primarily adolescents between the ages of 14-18, who mostly identified as women. Therefore, these results may not be representative of other genders, or other ages. Additionally, the data was collected from participants through social media. This means that those who do not spend time on social media would not have seen the survey. The age and gender of these participants depended heavily on the demographic of the social media account's followers. Also, the sample size of this study, 35 participants, may not be representative of a larger sample size. 


\subsection{Future Directions}

This study was conducted with an online survey. More experiments about masks and emotion recognition could be done in person to more accurately simulate reallife conditions. Additionally, future studies could investigate how emotions can be identified with masked facial expressions in addition to body language, tone of voice, etc.

Because emotion recognition is impaired by the usage of masks, there may be possible long-term effects on developing children. This could be studied in the future with young children who grew up during the pandemic. Hoemann and others proposed that infants learn emotion recognition by relating caregivers' facial expressions with behavior [10]. This would mean that if infants grew up in an environment that was constantly masked, the development of recognizing emotions may be affected.

\section{CONCLUSION}

This study found that the use of masks significantly affects the recognition of emotions. The recognition of fear, happiness, sadness, and disgust is affected by the use of masks, while the recognition of anger and neutrality is not affected significantly by the use of masks.

\section{REFERENCES}

[1] Schirmer, A., \& Adolphs, R. (2017). Emotion perception from face, voice, and touch: Comparisons and

convergence. Trends in Cognitive Sciences, 21(3), 216-228.

https://doi.org/10.1016\%2Fj.tics.2017.01.001

[2] Mandal, F. B. (2014). Nonverbal communication in humans. Journal of Human Behavior in the Social Environment, 24(4), 417-421. https://doi.org/10.1080/10911359.2013.831288

[3] Jack, R., \& Schyns, P. (2015). The human face as a dynamic tool for social communication. Current Biology, 25(14), R621-R634. https://doi.org/10.1016/j.cub.2015.05.052

[4] Wegrzyn, M., Vogt, M., Kireclioglu, B., Schneider, J., \& Kissler, J. (2017). Mapping the emotional face. how individual face parts contribute to successful emotion recognition. PLOS One, 12(5), e0177239. https://doi.org/10.1371/journal.pone.0177239

[5] Guo, K. (2012). Holistic gaze strategy to categorize facial expression of varying intensities. PLOS One, $7(8)$, e42585. https://doi.org/10.1371/journal.pone.0042585

[6] Kotsia, I., Buciu, I., \& Pitas, I. (2008). An analysis of facial expression recognition under partial facial image occlusion. Image and Vision Computing, 26(7), 1052-1067. https://doi.org/10.1016/j.imavis.2007.11.004

[7] Nestor, M. S., Fischer, D., \& Arnold, D. (2020). "Masking" our emotions: Botulinum toxin, facial expression, and well - being in the age of covid 19. Journal of Cosmetic Dermatology, 19(9), 21542160. https://doi.org/10.1111/jocd.13569

[8] Ebner, N. C., Riediger, M., \& Lindenberger, U. (2018). FACES: A database of facial expressions in young, middle-aged, and older women and men (publicly available datasets). Max Planck Society. Retrieved September 4, 2021, from https://faces.mpdl.mpg.de/imeji/collection/IXTdg7 21TwZwyZ8e

[9] Random.org. (1998). Random integer generator. Random.org. Retrieved September 4, 2021, from https://www.random.org/integers/?num $=1 \&$ min $=1$ $\& \max =2 \&$ col $=1 \&$ base $=10 \&$ format $=$ html $\&$ rnd $=$ ne $\mathrm{W}$

[10] Hoemann, K., Xu, F., \& Barrett, L. F. (2019). Emotion words, emotion concepts, and emotional development in children: A constructionist hypothesis. Developmental Psychology, 55(9), 1830-1849. https://doi.org/10.1037\%2Fdev0000686 